\title{
Hand dominance in early and established rheumatoid arthritis: evaluation by dynamometer, Ritchie index and musculoskeletal ultrasound: a cross sectional study
}

\author{
A.M. Elsaman', A. Sayed ${ }^{2}$, A.R. Radwan ${ }^{1}$ \\ ${ }^{1}$ Associate Professors of Rheumatology, Department of Rheumatology and Rehabilitation, \\ Sohag University Hospital, Sohag University, Sohag, Egypt; ${ }^{2}$ Consultant of Pain Management, \\ Armed Forces Specialized Center of Rehabilitation, Altaif, Kingdom of Saudi Arabia
}

\begin{abstract}
SUMMARY
Rheumatoid arthritis (RA) usually occurs as a symmetrical disease, which mainly affects the small joints of the hands and feet. The correlation of handedness with radiological changes shows significantly greater radiological changes in the dominant hand than in the non-dominant one. Additionally, the dominant hand is more severely affected in terms of strength, function and deformity. Our objective is to evaluate the influence of handedness on musculoskeletal ultrasound (US), Ritchie articular index (RAI) and digital dynamometer findings in patients with active RA (early, group B, vs. established, group A). A total number of 113 patients with established RA and 44 patients with early RA with active disease (DAS28-ESR >3.2) were included in the study. US assessments of both hands were performed to assess synovitis, tenosynovitis, and erosions. RAI was used to evaluate three joint groups in each hand. Handgrip strength was measured with a digital dynamometer. The US5 score showed that the dominant hand was more affected than the non-dominant one. This was significant in group A for the synovitis Power Doppler $(\mathrm{PD})$ mode $(\mathrm{p}=0.032)$ and tenosynovitis PD $(\mathrm{p}=0.005)$ scores, and in group B for synovitis Grey Scale (GS) mode $(\mathrm{p}<0.001)$, synovitis PD $(\mathrm{p}=0.037)$ and erosions ( $p=0.027)$ scores. RAI was significantly higher in the dominant hand $(p=0.013)$ in group $A$ and even greater in group $\mathrm{B}(\mathrm{p}=0.011)$. The dominant hand was stronger than the non-dominant hand in both groups. The dominant hand is generally affected in early RA. Subsequently, the disease tends to become more symmetrical with disease progression.
\end{abstract}

Key words: Rheumatoid arthritis; early, established; hand dominance; ultrasound.

Reumatismo, 2020; 72 (3): 131-144

\section{INTRODUCTION}

heumatoid arthritis (RA) is a common autoimmune disease. It affects about $1 \%$ of the population worldwide (1). It is a chronic inflammatory symmetrical disease which affects mainly small joints, especially at its onset (2).

Several previous studies have addressed the effect of handedness on RA progression. Most of them compared both hands in terms of radiological score, hand deformity, handgrip strength and Ritchie articular index (RAI) (3-5). These studies concluded that hand dominance has an impact on the disease course, and hypothesized that mechanical stress increase the disease plateau $(3,4)$, which is mainly seen in early RA $(5,6)$.

In many studies, RA disease activity and outcome were evaluated by ultrasound (US), which is a useful, noninvasive, rapid, painless bedside method preferred by most patients and physicians. It can detect early changes in bone (such as erosions) and soft tissues (such as synovitis and tenosynovitis) with high sensitivity. The 'US5 score' was proposed as a modification of the US7 score for hand evaluation in RA (7-12).
Corresponding author: Ahmed M. Elsaman Department of Rheumatology and Rehabilitation, Sohag University Hospital, Sohag University, Nile Street, Sohag City (Postal code 82524), Sohag Governorate, Egypt E-mail: ahmed_elsaman@med.sohag.edu.eg 
RAI is a summation of tenderness scores for joints calculated by applying pressure on the articular margins. This score is widely used for evaluation of RA activity (13). RAI is considered a standard method for comparing the dominant and non-dominant hands in RA (5).

The handgrip is usually affected by the activity of RA and its evaluation reflects to a great extent functional impairment in RA patients (14). Weakness is usually the result of muscle atrophy, joint pain, and stiffness. Hydraulic, pneumatic, and digital dynamometers are generally used for handgrip measurement (15).

In this study, we aimed to answer the following questions: i) how does disease symmetry change with disease progression? ii) does hand dominance constitute an important aspect of the clinical manifestations of $\mathrm{RA}$, and does it influence disease progression? iii) should both hands be included in imaging scores (i.e. US) or is it sufficient to examine one hand?

\section{MATERIALS AND METHODS}

Permission of publishing data related to the study participants was obtained. Personal and medical information was kept confidential and not made available to third parties. Patients with RA diagnosed on the basis of 2010 ACR/EULAR criteria (16) were recruited from the outpatient clinic of the rheumatology department, all of whom aged 18 or more. ESR was determined to calculate the DAS28, and patients with active disease (defined as DAS28-ESR >3.2) were included in the study (17). Patients with overlapping syndromes were excluded.

Patients were divided into two groups according to disease duration. Group A with established RA (disease duration $\geq 6$ months) and group B with early RA (disease duration $<6$ months) (18). The two groups were age- and sex-matched.

A descriptive cross-sectional study was carried out on 226 hands of 113 patients (six of them were left-handed) including 30 males and 83 females with established RA (group A), and 88 hands of 44 patients
( 2 of them were left-handed) with early RA (group B) including 16 males and 28 females. All patients in both groups were naïve for biologic therapies.

The dominant hand was defined as the hand that is used for handwriting or scissoring or daily activity $(5,6)$. Hand dominance in both groups was evaluated by US, RAI, and a dynamometer.

Musculoskeletal US was performed using a 7-12 MHz (General Electric Logic E, China) linear probe. Five joints [wrist, metacarpophalangeal joints (MCP) 2,3, proximal interphalangeal joints (PIP) 2,3] on both hands (bilateral 'US5 score'), which are part of the German US7 score, were assessed to evaluate synovitis, tenosynovitis and erosions in each hand (12). The US assessment included evaluation of synovitis, tenosynovitis, and erosions by grey scale (GS) and power Doppler (PD) mode (19). Synovitis and synovial/ tenosynovial vascularity were scored on a semiquantitative scale (grade 0-3) by power Doppler ultrasound (PDUS) (20). Synovitis (effusion and or synovial hypertrophy) was considered semi-quantitatively (0-3) by gray scale ultrasound (GSUS) (21). Tenosynovitis and erosions in GSUS were evaluated as being 0 , when absent, or 1, when present (12). All the joints included were examined from the palmar side, the dorsal side, and also the radial side of MCP II (only for erosion) (Figure $1)$. The $1^{\text {st }}$ and $3^{\text {rd }}$ author performed the US examination and both were blinded for clinical data. The $1^{\text {st }}$ author has 8 years of experience in the field of musculoskeletal US and performs 6 US examinations daily on average. The $3^{\text {rd }}$ author has 6 years of experience in the same field and carries out 6 US examinations daily on average. The interobserver Cohen's Kappa value was 0.73 , which means a good to excellent agreement between the two observers.

RAI is a score that is used for the assessment of degrees of joint tenderness in RA. It gives three grades for joint tenderness: grade 0 means no tenderness, grade 1 means that the patient complains of pain, grade 2 means that the patient complains of pain and winces, and grade 3 means 
that the patient complains of pain, winces and withdraws his or her hands. The joints evaluated by this score in our study included the wrist, MCP 1-5 joints (considered as one joint group), PIP 2-5 joints and interphalangeal (IP) joints (IP and PIP were considered as one joint group) in each hand (22). A minimum score of 0 and a maxi-
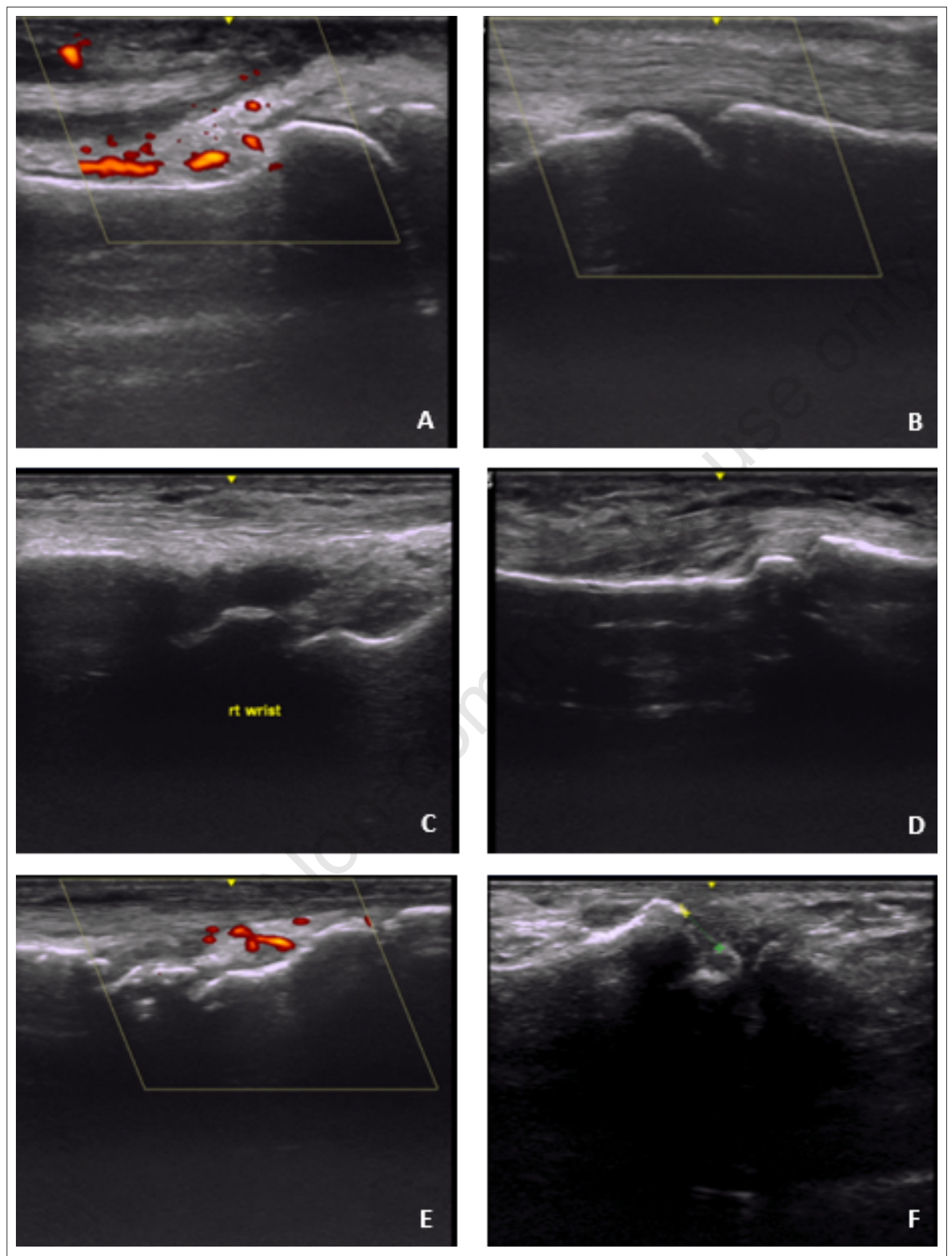

Figure 1 - Different features of the US5 score. A) MCP palmar sagittal scan with flexor tenosynovitis grade 2 by PDUS; B) MCP palmar sagital scan with grade 1 synovitis on GS and 0 on PDUS for synovitis and tenosynovitis; C) wrist palmar sagittal scan with grade 3 synovitis on GS; D) PIP palmar sagittal scan with synovitis grade 1 and tenosynovitis by GS; E) Wrist palmar longitudinal scan with grade 2 synovitis by PDUS; F) MCP palmar sagittal scan showing erosion. 
mum score of 9 for each hand was considered. Firm pressure was applied to the joint margin (enough to blanch the examiner's nail bed) (23).

Handgrip was measured using a baseline digital dynamometer (produced by Prohealth Care Company, New York; USA). During handgrip examination, the patient was standing with his back straight, shoulder slightly abducted, elbow fully extended and wrist extended. The display screen was facing the examiner. The physician requested the patient to squeeze as much as possible and then the examiner asked to compress harder and harder (24). The highest of three measures within a 2-minute interval (in order to avoid fatigue) was considered $(15,25)$. The dominant hand was tested first.

The US examination was done first, followed by the handgrip test and finally RAI. RAI was postponed to the last step in order not to affect the other two examinations. All three examinations were done on the same day.

A DAS28-ESR $>3.2$ was used as cut-off for the inclusion of RA patients with active disease. We excluded patients in remission or with low disease activity. RAI, hand grip and DAS28-ESR all were carried out by the second author, who was blinded for the US examination.

Data is presented as an arithmetic mean \pm SD as all of the quantitative data were normally distributed (Kolmogorov-Smirnov test). Student's t-test was used to compare means between two independent groups and the paired t-test was used to compare means of the same patient group between dominant and non-dominant hands. Comparisons of percentage qualitative data were tested using the Chi-square test or Fisher's exact test. Instead, for comparing percentages between the two hands in the same group, the McNemar Chi-square test was used. Correlations between two quantitative data were assessed using Pearson's correlation test. The Statistical Package for the Social Sciences (SPSS) software (version 22.0, SPSS Inc., Chicago, IL, USA) was used for all statistical analyses. A $p$ value $<0.05$ was considered significant.

\section{RESULTS}

The mean age of the patients was $39.73 \pm 10.11$ years in group A and $37.59 \pm 6.29$ years in group B. The male-to-female ratio was around 1:3 in group A and 1:2 in group B. The mean disease duration was $36.73 \pm 17.03$ months in group A and was 3.53 \pm 1.44 months in group B (Table I).

\section{Musculoskeletal ultrasound}

Regarding the US5 score, the mean scores of GS-US5 synovitis score, PD-US5 synovitis score, GS-US5 tenosynovitis score, PD-US5 tenosynovitis score and erosion sum score of 5 joints were 27.24 \pm 7.68 , $2.06 \pm 2.78, \quad 1.75 \pm 1.53, \quad 0.63 \pm 0.97$ and $1.94 \pm 2.07$ respectively in group $\mathrm{A}$, compared to $22.09 \pm 6.41,1.64 \pm 1.88,1.32 \pm 1.23$, $0.55 \pm 0.70$ and $0.93 \pm 1.19$ respectively in group B. The erosion and synovitis (GS only) scores were significantly higher in group A ( $\mathrm{p}=0.006$ and $<0.001$, respectively). No significant differences between the two groups were detected for the other components of the US5 score (Table I).

Among group $\mathrm{A}$, synovitis was the most common finding (found in $100 \%$ of cases, with different degrees) followed by tenosynovitis (found in more than $60 \%$ of cases), and lastly erosions (found in $58 \%$ of patients) (Tables II-IV). In group $\mathrm{B}$, synovitis was also the most common finding (found in $100 \%$ of cases, with different degrees) followed by tenosynovitis (found in 50\% of cases) and lastly erosions (found in around $45 \%$ of patients) (Tables II, III, V).

The comparison between the dominant and non-dominant hands regarding the US5 score in group A showed that the dominant hand was significantly more affected in the synovitis PD $(\mathrm{p}=0.032)$ and tenosynovitis PD $(p=0.003)$. In group $B$, the US5 score showed that the dominant hand was significantly more affected for synovitis GS $(\mathrm{p}<0.001)$, synovitis PD $(\mathrm{p}=0.037)$, and erosions $(\mathrm{p}=0.027)$ (Table VI).

Seropositive patients were more symmetrical, especially in group A. In group A, the US5 score showed non-significant differences between both hands among RF- 
Table I - Demographics, and clinical and sonographic comparison between the two study groups.

\begin{tabular}{|c|c|c|c|c|}
\hline & & $\begin{array}{c}\text { Group A } \\
\text { (Established RA) } \\
(\mathrm{N}=113)\end{array}$ & $\begin{array}{c}\text { Group B } \\
\text { (Early RA) } \\
\text { (N=44) }\end{array}$ & $P$ value \\
\hline Sex & Male & $30(26.5 \%)$ & $16(36.4 \%)$ & 0.225 \\
\hline & Female & $83(73.5 \%)$ & $28(63.6 \%)$ & \\
\hline Age & Mean (SD) & $39.73(10.11)$ & $37.59(6.29)$ & $0.113^{* *}$ \\
\hline & Median (range) & $37(25-62)$ & $36(25-53)$ & \\
\hline Disease duration (in months) & Mean (SD) & $36.73(17.03)$ & $3.53(1.44)$ & $<0.001^{* *}$ \\
\hline & Median (range) & $34(9-80)$ & $3.5(1.5-6)$ & \\
\hline RF & Positive & $95(84.1 \%)$ & $25(56.8 \%)$ & $<0.001$ \\
\hline Dominant hand & Right & $107(94.7 \%)$ & $42(95.5 \%)$ & $1.000^{*}$ \\
\hline & Left & $6(5.3 \%)$ & $2(4.5 \%)$ & \\
\hline DAS28 & Mean (SD) & $4.81(1.21)$ & $4.60(1.06)$ & $0.295^{\star *}$ \\
\hline & Moderate disease activity & $76(67.3 \%)$ & $31(70.5 \%)$ & 0.699 \\
\hline & High disease activity & $37(32.7 \%)$ & $13(29.5 \%)$ & 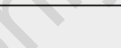 \\
\hline Ritchie, mean (SD) & Dominant hand & $4.98(1.65)$ & $4.77(1.82)$ & $0.488^{\star \star}$ \\
\hline & Non dominant hand & $4.55(1.63)$ & $3.93(1.56)$ & $0.032^{* *}$ \\
\hline & Total Richie & $9.53(2.71)$ & $8.70(2.66)$ & $0.087^{\star \star}$ \\
\hline Hand grip, mean (SD) & Dominant hand & $14.30(3.44)$ & $14.50(4.16)$ & $0.760^{\star \star}$ \\
\hline & Non dominant hand & $14.05(3.79)$ & $13.30(3.61)$ & $0.256^{\star *}$ \\
\hline US5 score items & & 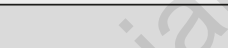 & & \\
\hline Synovitis, B mode, mean (SD) & Dominant hand & $13.90(4.64)$ & $12.32(3.81)$ & $0.046^{\star *}$ \\
\hline & Non dominant hand & $13.34(4.24)$ & $9.77(3.50)$ & $<0.001^{* *}$ \\
\hline & Both hands & $27.24(7.68)$ & $22.09(6.41)$ & $<0.001^{* *}$ \\
\hline Synovitis, PD, mean (SD) & Dominant hand & $1.27(2.44)$ & $1.09(1.67)$ & $0.861+$ \\
\hline & Non dominant hand & $0.79(0.87)$ & $0.55(0.63)$ & $0.181+$ \\
\hline & Both hands & $2.06(2.78)$ & $1.64(1.88)$ & $0.566+$ \\
\hline Tenosynovitis, B mode, mean & Dominant hand & $0.78(0.86)$ & $0.61(0.72)$ & $0.359+$ \\
\hline & Non dominant hand & $0.97(1.03)$ & $0.70(0.88)$ & $0.136+$ \\
\hline & Both hands & $1.75(1.53)$ & $1.32(1.23)$ & $0.135+$ \\
\hline Tenosynovitis, PD, mean (SD) & Dominant hand & $0.17(0.42)$ & $0.25(0.53)$ & $0.388+$ \\
\hline & Non dominant hand & $0.46(0.91)$ & $0.30(0.55)$ & $0.547+$ \\
\hline & Both hands & $0.63(0.97)$ & $0.55(0.70)$ & $0.876+$ \\
\hline Erosions, mean (SD) & Dominant hand & $0.98(1.36)$ & $0.64(0.94)$ & $0.226+$ \\
\hline & Non dominant hand & $0.96(1.09)$ & $0.30(0.55)$ & $<0.001+$ \\
\hline & Both hands & $1.94(2.07)$ & $0.93(1.19)$ & $0.006+$ \\
\hline Treatment & NSAIDs & $56(42.11 \%)$ & $21(47.73 \%)$ & 0.514 \\
\hline & Steroids & $26(19.55 \%)$ & $13(29.55 \%$ & 0.166 \\
\hline & MTX alone & $33(24.81 \%)$ & $18(40.91 \%)$ & 0.040 \\
\hline & $\mathrm{HCQ}$ alone & $1(0.75 \%)$ & $5(11.36 \%)$ & $0.004^{*}$ \\
\hline & LEF alone & $14(10.53 \%)$ & $9(20.45 \%)$ & 0.089 \\
\hline & $\mathrm{HCQ}+\mathrm{SSZ}$ & $5(3.76 \%)$ & $6(13.64 \%)$ & 0.046 \\
\hline & $\mathrm{MTX}+\mathrm{HCQ}$ & $22(16.54 \%)$ & $5(11.36 \%)$ & 0.407 \\
\hline & $\mathrm{MTX}+\mathrm{HCQ}+\mathrm{SSZ}$ & $12(9.02 \%)$ & 0 & $0.086^{*}$ \\
\hline & MTX + LEF & 46 (34.59\%) & $1(2.27 \%)$ & $<0.001$ \\
\hline
\end{tabular}

*Fisher's Exact test is used instead of Pearson Chi square.

**Student's t test was done to compare means between the two groups.

+Mann Whitney test was used in non-parametric data instead of Student's t test.

MTX: Methotrexate, HCQ: Hydroxychloroquine, SSZ: Sulfasalazine, LEF: Leflunomide. 
Table II - Comparison between dominant and non-dominant hand regarding Ritchie's index, hand grip and US5.

\begin{tabular}{|c|c|c|c|c|}
\hline & & $\begin{array}{l}\text { Dominant } \\
\text { hand }\end{array}$ & $\begin{array}{l}\text { Non- } \\
\text { dominant } \\
\text { hand }\end{array}$ & $P$ value \\
\hline \multirow[t]{3}{*}{ Ritchie, mean (SD) } & Group A (established RA) & $4.98(1.65)$ & $4.55(1.63)$ & 0.013 \\
\hline & Group B (Early RA) & $4.77(1.82)$ & $3.93(1.56)$ & 0.011 \\
\hline & All patients & $4.92(1.69)$ & $4.38(1.63)$ & $<0.001$ \\
\hline \multirow[t]{3}{*}{ Hand grip, mean (SD) } & Group A (established RA) & $14.30(3.44)$ & $14.05(3.79)$ & 0.486 \\
\hline & Group B (Early RA) & $14.50(4.16)$ & $13.30(3.61)$ & 0.045 \\
\hline & All patients & $14.36(3.64)$ & $13.84(3.74)$ & 0.092 \\
\hline \multicolumn{5}{|l|}{ US5 score items } \\
\hline \multirow[t]{3}{*}{ Synovitis (B mode) (score 0-33) } & Group (A) established RA & $13.90(4.64)$ & $13.34(4.24)$ & 0.181 \\
\hline & Group (B) early RA & $12.32(3.81)$ & $9.77(3.50)$ & $<0.001$ \\
\hline & Both groups & $13.46(4.47)$ & $12.34(4.34)$ & 0.001 \\
\hline \multirow[t]{3}{*}{ Synovitis (Power Doppler) (score 0-33) } & Group (A) established RA & $1.27(2.44)$ & $0.79(0.87)$ & 0.032 \\
\hline & Group (B) early RA & $1.09(1.67)$ & $0.55(0.63)$ & 0.037 \\
\hline & Both groups & $1.22(2.24)$ & $0.72(0.82)$ & 0.005 \\
\hline \multirow[t]{3}{*}{ Tenosynovitis (B mode) (Score 0-5) } & Group (A) established RA & $0.78(0.86)$ & $0.97(1.03)$ & 0.070 \\
\hline & Group (B) early RA & $0.61(0.72)$ & $0.70(0.88)$ & 0.561 \\
\hline & Both groups & $0.73(0.83)$ & $0.90(0.99)$ & 0.062 \\
\hline \multirow{3}{*}{$\begin{array}{l}\text { Tenosynovitis (Power Doppler) } \\
\text { (score 0-15) }\end{array}$} & Group (A) established RA & $0.17(0.42)$ & $0.46(0.91)$ & 0.003 \\
\hline & Group (B) early RA & $0.25(0.53)$ & $0.30(0.55)$ & 0.719 \\
\hline & Both groups & $0.190(0.45)$ & $0.41(0.82)$ & 0.005 \\
\hline \multirow[t]{3}{*}{ Erosions (score 0-9) } & Group (A) established RA & $0.98(1.36)$ & $0.96(1.09)$ & 0.833 \\
\hline & Group (B) early RA & $0.63(0.94)$ & $0.30(0.55)$ & 0.027 \\
\hline & Both groups & $0.89(1.27)$ & $0.77(1.01)$ & 0.254 \\
\hline
\end{tabular}

Paired t test was used in all of the above comparisons.

positive patients. A significant difference among RF-negative patients was seen only for PD tenosynovitis US5 score ( $\mathrm{p}=0.014)$. In group B, the US5 score showed a significant difference between both hands among RF positive patients for the synovitis GS score $(\mathrm{p}=0.006)$. Furthermore, a significant difference was present in RF-negative patients for synovitis (only GS) $(\mathrm{p}=0.001)$, tenosynovitis (only GS) $(\mathrm{p}=0.031)$, and erosion US5 scores $(\mathrm{p}=0.049)$. Asymmetry was more often found in the wrist followed by MCP and PIP joints in group A. Symmetry was measured qualitatively by comparing joints affected in both hands, and quantitatively by comparing the US5 score between both hands.

The DAS28 correlated with the compo- nents of the US5 score, but there was no significant difference between the two groups. Only the erosion score in group $\mathrm{B}$ was significantly higher in patients with a high DAS28 (compared to moderate DAS28) $(\mathrm{p}=0.012)$.

\section{RAI}

In group A, the RAI was significantly higher in the dominant hand compared to the nondominant hand $(\mathrm{p}=0.013)$, and in group $\mathrm{B}$, the difference was even more significant ( $p=0.011$ ). As to the comparison of RAI between the established and early RA groups, it was higher in group A. However, the difference between both groups was significant in the non-dominant hand and not significant in the dominant hand (Table VI). 
Table III - Frequency of positive findings by US5 score in the dominant hand according to disease duration and joint.

\begin{tabular}{|c|c|c|c|c|}
\hline & & Group A & Group B & $P$ value \\
\hline \multirow{12}{*}{$\begin{array}{l}\text { Synovitis } \\
\text { (B mode) }\end{array}$} & Wrist dorso-median & $103(91.2 \%)$ & $38(86.4 \%)$ & $0.268^{*}$ \\
\hline & Wrist dorso-ulnar & $83(73.5 \%)$ & $30(68.2 \%)$ & 0.509 \\
\hline & $2^{\text {nd }}$ MCP dorsal & 84 (74.3\%) & $31(70.5 \%)$ & 0.622 \\
\hline & $3^{\text {rd }}$ MCP dorsal & 79 (69.9\%) & $29(65.9 \%)$ & 0.627 \\
\hline & $2^{\text {nd }}$ PIP dorsal & 93 (82.3\%) & $36(81.8 \%)$ & 0.943 \\
\hline & $3^{\text {rd }}$ PIP dorsal & $91(80.5 \%)$ & $37(84.1 \%)$ & 0.606 \\
\hline & Wrist palmo-median & $87(77 \%)$ & 32 (72.7\%) & 0.575 \\
\hline & $2^{\text {nd }} \mathrm{MCP}$ palmar & $73(64.6 \%)$ & $28(63.6 \%)$ & 0.910 \\
\hline & $3^{\text {rd }}$ MCP palmar & 72 (63.7\%) & $26(59.1 \%)$ & 0.591 \\
\hline & $2^{\text {nd }}$ PIP palmar & $81(71.7 \%)$ & $30(68.2 \%)$ & 0.665 \\
\hline & $3^{\text {rd }}$ PIP palmar & 77 (68.1\%) & $30(68.2 \%)$ & 0.996 \\
\hline & All & $113(100 \%)$ & $44(100 \%)$ & - \\
\hline \multirow{12}{*}{$\begin{array}{l}\text { Synovitis } \\
\text { (Power Doppler) }\end{array}$} & Wrist dorso-median & $42(37.1 \%)$ & $13(29.6 \%)$ & 0.369 \\
\hline & Wrist dorso-ulnar & $8(7.1 \%)$ & $4(9.1 \%)$ & $0.740^{*}$ \\
\hline & $2^{\text {nd }}$ MCP dorsal & $6(5.3 \%)$ & $4(9.1 \%)$ & $0.468^{*}$ \\
\hline & $3^{\text {rd }}$ MCP dorsal & $4(3.5 \%)$ & $1(2.3 \%)$ & $1.000^{*}$ \\
\hline & $2^{\text {nd }} P I P$ dorsal & $14(12.4 \%)$ & $4(9.1 \%)$ & 0.560 \\
\hline & $3^{\text {rd }}$ PIP dorsal & $4(3.5 \%)$ & $2(4.5 \%)$ & $0.673^{*}$ \\
\hline & Wrist palmo-median & $7(6.3 \%)$ & $4(9.1 \%)$ & $0.503^{*}$ \\
\hline & 2nd MCP palmar & $6(5.3 \%)$ & $3(6.8 \%)$ & $0.711^{\star}$ \\
\hline & $3^{\text {rd }}$ MCP palmar & $4(3.5 \%)$ & $2(4.5 \%)$ & $0.673^{*}$ \\
\hline & $2^{\text {nd }} \mathrm{PIP}$ palmar & $10(8.8 \%)$ & $4(9.1 \%)$ & $1.000^{*}$ \\
\hline & $3^{\text {rd }}$ PIP palmar & $2(1.8 \%)$ & 0 & $1.000^{*}$ \\
\hline & All & $60(53.1 \%)$ & $24(54.5 \%)$ & 0.870 \\
\hline \multirow[t]{6}{*}{ Tenosynovitis (B mode) } & Wrist dorso-median & $20(17.7 \%)$ & $6(13.6 \%)$ & 0.539 \\
\hline & Wrist dorso-ulnar & $44(38.9 \%)$ & $10(22.7 \%)$ & 0.055 \\
\hline & Wrist palmo-median & $8(7.1 \%)$ & $4(9.1 \%)$ & $0.740^{*}$ \\
\hline & $2^{\text {nd }} \mathrm{MCP}$ palmar & $6(5.3 \%)$ & $2(4.5 \%)$ & $1.000^{*}$ \\
\hline & $3^{\text {rd }}$ MCP palmar & $10(8.8 \%)$ & $5(11.4 \%)$ & $0.763^{*}$ \\
\hline & All & $59(52.2 \%)$ & $22(50 \%)$ & 0.803 \\
\hline \multirow{6}{*}{$\begin{array}{l}\text { Tenosynovitis (Power } \\
\text { Doppler) }\end{array}$} & Wrist dorso-median & $7(6.2 \%)$ & $3(6.8 \%)$ & $1.000^{*}$ \\
\hline & Wrist dorso-ulnar & $8(7.1 \%)$ & $4(9.1 \%)$ & $0.740^{*}$ \\
\hline & Wrist palmo-median & $2(1.8 \%)$ & $2(4.5 \%)$ & $0.313^{*}$ \\
\hline & 2nd MCP palmar & 0 & $1(2.3 \%)$ & $0.280^{*}$ \\
\hline & 3rd MCP palmar & 0 & 0 & - \\
\hline & All & $17(15 \%)$ & $9(20.5 \%)$ & 0.413 \\
\hline \multirow[t]{10}{*}{ Erosions } & $2^{\text {nd }} \mathrm{MCP}$ dorsal & $26(23 \%)$ & $9(20.5 \%)$ & 0.730 \\
\hline & $2^{\text {nd }} \mathrm{MCP}$ radial & 49 (43.4\%) & $7(15.9 \%)$ & 0.001 \\
\hline & $3^{\text {rd }}$ MCP dorsal & $8(7.1 \%)$ & $2(4.5 \%)$ & $0.727^{*}$ \\
\hline & $2^{\text {nd }}$ PIP dorsal & $6(5.3 \%)$ & $3(6.8 \%)$ & $0.711^{*}$ \\
\hline & $3^{\text {rd }}$ PIP dorsal & $4(3.5 \%)$ & $2(4.5 \%)$ & $0.673^{*}$ \\
\hline & $2^{\text {nd }} \mathrm{MCP}$ palmar & $7(6.2 \%)$ & $2(4.5 \%)$ & $1.000^{*}$ \\
\hline & $3^{\text {rd }}$ MCP palmar & $4(3.5 \%)$ & $1(2.3 \%)$ & $1.000^{*}$ \\
\hline & $2^{\text {nd }} P I P$ palmar & $4(3.5 \%)$ & $1(2.3 \%)$ & $1.000^{*}$ \\
\hline & $3^{\text {rd }}$ PIP palmar & $3(2.7 \%)$ & $1(2.3 \%)$ & $1.000^{*}$ \\
\hline & All & $56(49.6 \%)$ & $20(45.5 \%)$ & 0.644 \\
\hline
\end{tabular}

*Fisher exact test was used instead of Pearson chi square. 
Table IV - Frequency of positive findings by US5 score in group A according to the involved joint.

\begin{tabular}{|c|c|c|c|c|}
\hline \multirow{13}{*}{$\begin{array}{l}\text { Synovitis } \\
\text { (B mode) }\end{array}$} & & Dominant hand & Non dominant hand & $P$ value \\
\hline & Wrist dorso-median & $103(91.2 \%)$ & $103(91.2 \%)$ & 1.000 \\
\hline & Wrist dorso-ulnar & 83 (73.5\%) & $84(74.3 \%)$ & 0.879 \\
\hline & $2^{\text {nd }}$ MCP dorsal & $84(74.3 \%)$ & $71(62.8 \%)$ & 0.062 \\
\hline & $3^{\text {rd }}$ MCP dorsal & $79(69.9 \%)$ & $83(73.5 \%)$ & 0.555 \\
\hline & $2^{\text {nd }}$ PIP dorsal & $93(82.3 \%)$ & $99(87.6 \%)$ & 0.264 \\
\hline & $3^{\text {rd }}$ PIP dorsal & $91(80.5 \%)$ & $96(85 \%)$ & 0.379 \\
\hline & Wrist palmo-median & 87 (77\%) & $92(81.4 \%)$ & 0.412 \\
\hline & $2^{\text {nd }} \mathrm{MCP}$ palmar & 73 (64.6\%) & 78 (69\%) & 0.480 \\
\hline & $3^{\text {rd }}$ MCP palmar & $72(63.7 \%)$ & $66(58.4 \%)$ & 0.413 \\
\hline & $2^{\text {nd }}$ PIP palmar & $81(71.7 \%)$ & $86(76.1 \%)$ & 0.449 \\
\hline & $3^{\text {rd }}$ PIP palmar & 77 (68.1\%) & $68(60.2 \%)$ & 0.212 \\
\hline & All & $113(100 \%)$ & $113(100 \%)$ & 1.000 \\
\hline \multirow{12}{*}{$\begin{array}{l}\text { Synovitis } \\
\text { (Power Doppler) }\end{array}$} & Wrist dorso-median & 42 (37.1\%) & $50(44.2 \%)$ & 0.279 \\
\hline & Wrist dorso-ulnar & $8(7.1 \%)$ & $10(8.8 \%)$ & 0.623 \\
\hline & $2^{\text {nd }} \mathrm{MCP}$ dorsal & $6(5.3 \%)$ & 0 & 0.013 \\
\hline & $3^{\text {rd }}$ MCP dorsal & $4(3.5 \%)$ & 0 & 0.044 \\
\hline & $2^{\text {nd }}$ PIP dorsal & $14(12.4 \%)$ & $6(5.3 \%)$ & 0.061 \\
\hline & $3^{\text {rd }}$ PIP dorsal & $4(3.5 \%)$ & 0 & 0.044 \\
\hline & Wrist palmo-median & $7(6.3 \%)$ & $3(2.7 \%)$ & 0.196 \\
\hline & $2^{\text {nd }} \mathrm{MCP}$ palmar & $6(5.3 \%)$ & $3(2.7 \%)$ & 0.308 \\
\hline & $3^{\text {rd }}$ MCP palmar & $4(3.5 \%)$ & $3(2.7 \%)$ & 0.701 \\
\hline & $2^{\text {nd }}$ PIP palmar & $10(8.8 \%)$ & 0 & 0.001 \\
\hline & $3^{\text {rd }}$ PIP palmar & $2(1.8 \%)$ & 0 & 0.155 \\
\hline & All & $60(53.1 \%)$ & 62 (54.9\%) & 0.790 \\
\hline \multirow{6}{*}{$\begin{array}{l}\text { Tenosynovitis } \\
\text { (B mode) }\end{array}$} & Wrist dorso-median & $20(17.7 \%)$ & $26(23 \%)$ & 0.321 \\
\hline & Wrist dorso-ulnar & $44(38.9 \%)$ & $40(35.4 \%)$ & 0.582 \\
\hline & Wrist palmo-median & $8(7.1 \%)$ & $15(13.3 \%)$ & 0.123 \\
\hline & $2^{\text {nd }} \mathrm{MCP}$ palmar & $6(5.3 \%)$ & $17(15 \%)$ & 0.016 \\
\hline & $3^{\text {rd }}$ MCP palmar & $10(8.8 \%)$ & 0 & 0.001 \\
\hline & All & $59(52.2 \%)$ & 69 (61.1\%) & 0.179 \\
\hline \multirow{6}{*}{$\begin{array}{l}\text { Tenosynovitis } \\
\text { (Power Doppler) }\end{array}$} & Wrist dorso-median & $7(6.2 \%)$ & $14(12.4 \%)$ & 0.109 \\
\hline & Wrist dorso-ulnar & $8(7.1 \%)$ & $14(12.4 \%)$ & 0.178 \\
\hline & Wrist palmo-median & $2(1.8 \%)$ & $5(4.4 \%)$ & 0.249 \\
\hline & $2^{\text {nd }} \mathrm{MCP}$ palmar & 0 & $5(4.4 \%)$ & 0.024 \\
\hline & $3^{\text {rd }}$ MCP palmar & 0 & 0 & 1.000 \\
\hline & All & $17(15 \%)$ & $32(28.3 \%)$ & 0.015 \\
\hline \multirow[t]{10}{*}{ Erosions } & $2^{\text {nd }} M C P$ dorsal & $26(23 \%)$ & $20(17.7 \%)$ & 0.321 \\
\hline & $2^{\text {nd }}$ MCP radial & $49(43.4 \%)$ & $47(41.6 \%)$ & 0.788 \\
\hline & $3^{\text {rd }}$ MCP dorsal & $8(7.1 \%)$ & $3(2.7 \%)$ & 0.122 \\
\hline & $2^{\text {nd }}$ PIP dorsal & $6(5.3 \%)$ & $6(5.3 \%)$ & 1.000 \\
\hline & $3^{\text {rd }}$ PIP dorsal & $4(3.5 \%)$ & $4(3.5 \%)$ & 1.000 \\
\hline & $2^{\text {nd }}$ MCP palmar & $7(6.2 \%)$ & $13(11.5 \%)$ & 0.160 \\
\hline & $3^{\text {rd }}$ MCP palmar & $4(3.5 \%)$ & $7(6.2 \%)$ & 0.354 \\
\hline & $2^{\text {nd }}$ PIP palmar & $4(3.5 \%)$ & 0 & 0.044 \\
\hline & $3^{\text {rd }}$ PIP palmar & $3(2.7 \%)$ & $8(7.1 \%)$ & 0.122 \\
\hline & All & $56(49.6 \%)$ & $66(58.4 \%)$ & 0.182 \\
\hline
\end{tabular}

McNemar chi square test was used. 
Table V - Frequency of positive findings by US5 score in group B according to the involved joint.

\begin{tabular}{|c|c|c|c|c|}
\hline \multirow{3}{*}{$\begin{array}{l}\text { Synovitis } \\
\text { (B mode) }\end{array}$} & & Dominant hand & Non dominant hand & $P$ value \\
\hline & Wrist dorso-median & $38(86.4 \%)$ & $35(79.5 \%)$ & 0.395 \\
\hline & Wrist dorso-ulnar & $30(68.2 \%)$ & $18(40.9 \%)$ & 0.010 \\
\hline \multirow{10}{*}{ (B mode) } & $2^{\text {nd }}$ MCP dorsal & $31(70.5 \%)$ & $20(45.5 \%)$ & 0.018 \\
\hline & $3^{\text {rd }}$ MCP dorsal & $29(65.9 \%)$ & $28(63.6 \%)$ & 0.823 \\
\hline & $2^{\text {nd }}$ PIP dorsal & $36(81.8 \%)$ & $34(77.3 \%)$ & 0.597 \\
\hline & $3^{\text {rd }}$ PIP dorsal & $37(84.1 \%)$ & 35 (79.5\%) & 0.580 \\
\hline & Wrist palmo-median & $32(72.7 \%)$ & $24(54.5 \%)$ & 0.076 \\
\hline & $2^{\text {nd }}$ MCP palmar & $28(63.6 \%)$ & $26(59.1 \%)$ & 0.661 \\
\hline & $3^{\text {rd }}$ MCP palmar & $26(59.1 \%)$ & $24(54.5 \%)$ & 0.667 \\
\hline & $2^{\text {nd }}$ PIP palmar & $30(68.2 \%)$ & 31 (70.5\%) & 0.818 \\
\hline & $3^{\text {rd }}$ PIP palmar & $30(68.2 \%)$ & $28(63.6 \%)$ & 0.653 \\
\hline & All & $44(100 \%)$ & $44(100 \%)$ & 1.000 \\
\hline \multirow{12}{*}{$\begin{array}{l}\text { Synovitis } \\
\text { (Power Doppler) }\end{array}$} & Wrist dorso-median & $13(29.6 \%)$ & $16(35.4 \%)$ & 0.496 \\
\hline & Wrist dorso-ulnar & $4(9.1 \%)$ & $1(2.3 \%)$ & 0.167 \\
\hline & $2^{\text {nd }}$ MCP dorsal & $4(9.1 \%)$ & $1(2.3 \%)$ & 0.167 \\
\hline & $3^{\text {rd }}$ MCP dorsal & $1(2.3 \%)$ & 0 & 0.315 \\
\hline & $2^{\text {nd }}$ PIP dorsal & $4(9.1 \%)$ & $2(4.5 \%)$ & 0.398 \\
\hline & $3^{\text {rd }}$ PIP dorsal & $2(4.5 \%)$ & 0 & 0.153 \\
\hline & Wrist palmo-median & $4(9.1 \%)$ & $1(2.3 \%)$ & 0.167 \\
\hline & $2^{\text {nd }} \mathrm{MCP}$ palmar & $3(6.8 \%)$ & $1(2.3 \%)$ & 0.167 \\
\hline & $3^{\text {rd }}$ MCP palmar & $2(4.5 \%)$ & 0 & 0.153 \\
\hline & $2^{\text {nd }}$ PIP palmar & $4(9.1 \%)$ & 0 & 0.041 \\
\hline & $3^{\text {rd }}$ PIP palmar & 0 & $1(2.3 \%)$ & 0.315 \\
\hline & All & $24(54.5 \%)$ & $21(47.7 \%)$ & 0.522 \\
\hline \multirow{6}{*}{$\begin{array}{l}\text { Tenosynovitis } \\
\text { (B mode) }\end{array}$} & Wrist dorso-median & $6(13.6 \%)$ & $9(20.5 \%)$ & 0.395 \\
\hline & Wrist dorso-ulnar & $10(22.7 \%)$ & $11(25 \%)$ & 0.802 \\
\hline & Wrist palmo-median & $4(9.1 \%)$ & $2(4.5 \%)$ & 0.398 \\
\hline & $2^{\text {nd }} \mathrm{MCP}$ palmar & $2(4.5 \%)$ & $7(15.9 \%)$ & 0.079 \\
\hline & $3^{\text {rd }}$ MCP palmar & $5(11.4 \%)$ & 0 & 0.021 \\
\hline & All & $22(50 \%)$ & $21(47.7 \%)$ & 0.832 \\
\hline \multirow{6}{*}{$\begin{array}{l}\text { Tenosynovitis } \\
\text { (Power Doppler) }\end{array}$} & Wrist dorso-median & $3(6.8 \%)$ & $3(6.8 \%)$ & 1.000 \\
\hline & Wrist dorso-ulnar & $4(9.1 \%)$ & $7(15.9 \%)$ & 0.336 \\
\hline & Wrist palmo-median & $2(4.5 \%)$ & $2(4.5 \%)$ & 1.000 \\
\hline & $2^{\text {nd }} M C P$ palmar & $1(2.3 \%)$ & $1(2.3 \%)$ & 1.000 \\
\hline & $3^{\text {rd }}$ MCP palmar & 0 & 0 & 1.000 \\
\hline & All & $9(20.5 \%)$ & $11(25 \%)$ & 0.611 \\
\hline \multirow[t]{10}{*}{ Erosions } & $2^{\text {nd }} \mathrm{MCP}$ dorsal & $9(20.5 \%)$ & $2(4.5 \%)$ & 0.024 \\
\hline & $2^{\text {nd }} M C P$ radial & $7(15.9 \%)$ & $6(13.6 \%)$ & 0.764 \\
\hline & $3^{\text {rd }}$ MCP dorsal & $2(4.5 \%)$ & $1(2.3 \%)$ & 0.557 \\
\hline & $2^{\text {nd }}$ PIP dorsal & $3(6.8 \%)$ & $1(2.3 \%)$ & 0.306 \\
\hline & $3^{\text {rd }}$ PIP dorsal & $2(4.5 \%)$ & 0 & 0.153 \\
\hline & $2^{\text {nd }} \mathrm{MCP}$ palmar & $2(4.5 \%)$ & $1(2.3 \%)$ & 0.557 \\
\hline & $3^{\text {rd }}$ MCP palmar & $1(2.3 \%)$ & $1(2.3 \%)$ & 1.000 \\
\hline & $2^{\text {nd }}$ PIP palmar & $1(2.3 \%)$ & 0 & 0.315 \\
\hline & $3^{\text {rd }}$ PIP palmar & $1(2.3 \%)$ & $1(2.3 \%)$ & 1.000 \\
\hline & All & $20(45.5 \%)$ & $11(25.0 \%)$ & 0.045 \\
\hline
\end{tabular}

McNemar chi square test was used. 
Table VI - Frequency of positive findings by US5 score in the early RA group according to the involved joint.

\begin{tabular}{|c|c|c|c|c|}
\hline & & Dominant hand & Non dominant hand & $P$ value \\
\hline \multirow{12}{*}{$\begin{array}{l}\text { Synovitis } \\
\text { (B mode) }\end{array}$} & Wrist dorso-median & $38(86.4 \%)$ & $35(79.5 \%)$ & 0.395 \\
\hline & Wrist dorso-ulnar & 30 (68.2\%) & $18(40.9 \%)$ & 0.010 \\
\hline & $2^{\text {nd }}$ MCP dorsal & $31(70.5 \%)$ & $20(45.5 \%)$ & 0.018 \\
\hline & $3^{\text {rd }}$ MCP dorsal & $29(65.9 \%)$ & $28(63.6 \%)$ & 0.823 \\
\hline & $2^{\text {nd }}$ PIP dorsal & 36 (81.8\%) & $34(77.3 \%)$ & 0.597 \\
\hline & $3^{\text {rd }}$ PIP dorsal & $37(84.1 \%)$ & $35(79.5 \%)$ & 0.580 \\
\hline & Wrist palmo-median & $32(72.7 \%)$ & $24(54.5 \%)$ & 0.076 \\
\hline & $2^{\text {nd }}$ MCP palmar & $28(63.6 \%)$ & 26 (59.1\%) & 0.661 \\
\hline & $3^{\text {rd }}$ MCP palmar & $26(59.1 \%)$ & $24(54.5 \%)$ & 0.667 \\
\hline & $2^{\text {nd }}$ PIP palmar & $30(68.2 \%)$ & $31(70.5 \%)$ & 0.818 \\
\hline & $3^{\text {rd }}$ PIP palmar & $30(68.2 \%)$ & $28(63.6 \%)$ & 0.653 \\
\hline & All & $44(100 \%)$ & $44(100 \%)$ & 1.000 \\
\hline \multirow{12}{*}{$\begin{array}{l}\text { Synovitis } \\
\text { (Power Doppler) }\end{array}$} & Wrist dorso-median & $13(29.6 \%)$ & $16(35.4 \%)$ & 0.496 \\
\hline & Wrist dorso-ulnar & $4(9.1 \%)$ & $1(2.3 \%)$ & 0.167 \\
\hline & $2^{\text {nd }}$ MCP dorsal & $4(9.1 \%)$ & $1(2.3 \%)$ & 0.167 \\
\hline & $3^{\text {rd }}$ MCP dorsal & $1(2.3 \%)$ & 0 & 0.315 \\
\hline & $2^{\text {nd }}$ PIP dorsal & $4(9.1 \%)$ & $2(4.5 \%)$ & 0.398 \\
\hline & $3^{\text {rd }}$ PIP dorsal & $2(4.5 \%)$ & 0 & 0.153 \\
\hline & Wrist palmo-median & $4(9.1 \%)$ & $1(2.3 \%)$ & 0.167 \\
\hline & $2^{\text {nd }} \mathrm{MCP}$ palmar & $3(6.8 \%)$ & $1(2.3 \%)$ & 0.167 \\
\hline & $3^{\text {rd }} \mathrm{MCP}$ palmar & $2(4.5 \%)$ & 0 & 0.153 \\
\hline & $2^{\text {nd }}$ PIP palmar & $4(9.1 \%)$ & 0 & 0.041 \\
\hline & $3^{\text {rd }}$ PIP palmar & 0 & $1(2.3 \%)$ & 0.315 \\
\hline & All & $24(54.5 \%)$ & $21(47.7 \%)$ & 0.522 \\
\hline \multirow{6}{*}{$\begin{array}{l}\text { Tenosynovitis } \\
\text { (B mode) }\end{array}$} & Wrist dorso-median & $6(13.6 \%)$ & $9(20.5 \%)$ & 0.395 \\
\hline & Wrist dorso-ulnar & $10(22.7 \%)$ & $11(25 \%)$ & 0.802 \\
\hline & Wrist palmo-median & $4(9.1 \%)$ & $2(4.5 \%)$ & 0.398 \\
\hline & $2^{\text {nd }}$ MCP palmar & $2(4.5 \%)$ & $7(15.9 \%)$ & 0.079 \\
\hline & $3^{\text {rd }}$ MCP palmar & $5(11.4 \%)$ & 0 & 0.021 \\
\hline & All & $22(50 \%)$ & $21(47.7 \%)$ & 0.832 \\
\hline \multirow{6}{*}{$\begin{array}{l}\text { Tenosynovitis } \\
\text { (Power Doppler) }\end{array}$} & Wrist dorso-median & $3(6.8 \%)$ & $3(6.8 \%)$ & 1.000 \\
\hline & Wrist dorso-ulnar & $4(9.1 \%)$ & $7(15.9 \%)$ & 0.336 \\
\hline & Wrist palmo-median & $2(4.5 \%)$ & $2(4.5 \%)$ & 1.000 \\
\hline & $2^{\text {nd }} \mathrm{MCP}$ palmar & $1(2.3 \%)$ & $1(2.3 \%)$ & 1.000 \\
\hline & $3^{\text {rd }}$ MCP palmar & 0 & 0 & 1.000 \\
\hline & All & $9(20.5 \%)$ & $11(25 \%)$ & 0.611 \\
\hline \multirow[t]{10}{*}{ Erosions } & $2^{\text {nd }}$ MCP dorsal & $9(20.5 \%)$ & $2(4.5 \%)$ & 0.024 \\
\hline & $2^{\text {nd }} \mathrm{MCP}$ radial & $7(15.9 \%)$ & $6(13.6 \%)$ & 0.764 \\
\hline & $3^{\text {rd }}$ MCP dorsal & $2(4.5 \%)$ & $1(2.3 \%)$ & 0.557 \\
\hline & $2^{\text {nd }}$ PIP dorsal & $3(6.8 \%)$ & $1(2.3 \%)$ & 0.306 \\
\hline & $3^{\text {rd }}$ PIP dorsal & $2(4.5 \%)$ & 0 & 0.153 \\
\hline & $2^{\text {nd }}$ MCP palmar & $2(4.5 \%)$ & $1(2.3 \%)$ & 0.557 \\
\hline & $3^{\text {rd }}$ MCP palmar & $1(2.3 \%)$ & $1(2.3 \%)$ & 1.000 \\
\hline & $2^{\text {nd }}$ PIP palmar & $1(2.3 \%)$ & 0 & 0.315 \\
\hline & $3^{\text {rd }}$ PIP palmar & $1(2.3 \%)$ & $1(2.3 \%)$ & 1.000 \\
\hline & All & 20 (45.5\%) & $11(25.0 \%)$ & 0.045 \\
\hline
\end{tabular}

McNemar chi square test was used. 


\section{Handgrip}

The dominant hand was always stronger than the non-dominant hand in both groups. However, the difference was not significant in the established RA group ( $\mathrm{p}=0.486$ ), but it was significant in the early RA group $(\mathrm{p}=0.045)$ (Table VI).

\section{Comparison of the three modalities}

Using Pearson Correlation statistics to estimate the correlation between the three modalities (US5, RAI and hand grip) in the dominant hand and the non-dominant hand, the following results were obtained.

1. The correlations between US5 and RAI in the dominant hand were significant in group A for synovitis GS $(r=251$, $\mathrm{p}=0.007)$ and tenosynovitis $\mathrm{PD}(\mathrm{r}=0.262$, $\mathrm{p}=0.005$ ), while they were significant in group B for synovitis (GS) ( $\mathrm{r}=0.616$, $\mathrm{p}<0.001)$, tenosynovitis (PD) $(\mathrm{r}=0.324$, $\mathrm{p}=0.032)$ and erosions $(\mathrm{r}=0.345$, $\mathrm{p}=0.022)$. However, in the non-dominant hand, the correlations were non-significant in group A, whereas they were was significant in group $\mathrm{B}$ only for synovitis GS ( $r=0.312, p=0.039)$.

2. The correlations between US5 and hand grip in the dominant hand were non-significant in group A with the exception of synovitis GS $(\mathrm{r}=-0.263, \mathrm{p}=0.005)$ and synovitis $\mathrm{PD}(\mathrm{r}=-0.342, \mathrm{p}=0.001)$. In group $\mathrm{B}$, the correlations were significant for synovitis $G S(r=-0.295, p=0.050)$, synovitis $\mathrm{PD} \quad(\mathrm{r}=-0.413, \mathrm{p}=0.005)$, tenosynovitis PD $(\mathrm{r}=-0.267, \mathrm{p}=0.049)$ and erosions $(\mathrm{r}=-0.297, \mathrm{p}=0.039)$. In the non-dominant hand, all correlations were non-significant.

3. In group $\mathrm{A}$, the correlations between RAI and handgrip were $r=-334$, $(\mathrm{p}=0.001)$ in the dominant hand and $\mathrm{r}=$ $-0.374,(p<0.001)$ in the non-dominant hand, whereas in group B the correlations were $r=-0.484,(p<0.001)$ in the dominant hand and $\mathrm{r}=-0.510(\mathrm{p}<0.001)$ in the non-dominant hand.

\section{DISCUSSION}

US is gaining increasing importance in the field of early diagnosis and follow-up of disease activity in RA patients. Several scores have been developed for the assessment of RA activity by US $(21,26)$. Some scores included only one hand. Indeed, others included both hands, but the examination times recorded by this scores were too long, ranging from 20 to 70 minutes (27, 28 ) or they did not include a thorough examination of both hands (29).

There is no agreement in the literature on the effect of handedness on disease activity. Some state that the dominant hand is more affected; others maintain that the difference is not significant. This controversy could be attributed to the inclusion of patients with different disease durations $(3,4$, $7,8)$.

Some papers in the literature concluded that the difference is not significant and the disease is symmetrical in nature $(7,8)$ and even asymptomatic joints may have arthritis (9). In addition, one study in established RA showed no difference between the dominant hand and non-dominant hand in terms of joint space width (10).

The disease duration varied widely among all these studies. Furthermore, many of them did not clearly define it. US scores used for follow-up examinations of RA patients consider only the examination of the clinically dominant hand (i.e. for tenderness and swelling) (11). Since controversy has been raised about hand dominance in RA, further research is needed in this field. Our study was designed to compare hand dominance by detailed US examinations using the US5 score of both hands assessing synovitis, tenosynovitis, and erosions, and comparing US findings to RAI and hand grip strength.

We found that the commonest sonographic finding was synovitis followed by tenosynovitis and erosions in both groups, as was also emphasized by Mendonça et al (30). The sample size in that study is relatively small (only 39 participants), because the target was early arthritis. Disease duration at the beginning of the study ranged between 3 months and 2 years. They considered ACR 1987 criteria for diagnosis and the presence of a single synovitis by US as an entry criterion for all participants. The 
mean DAS was 4, which indicates inclusion of relatively active patients. The evaluation included conventional radiography and US assessment, by the modified US 7 score, measuring joint swelling and tenderness. The mean age of the participants was older than the mean age in our study. As to pain, the right wrist, MCP 2-5, PIP 3 and 5 had more pain, but the difference was not significant for most of these locations. For swelling, right wrist, MCP 2-4, and PIP 2, 3 , and 5 were more affected. For GS, right MCP 2 and MCP 3 were more significantly involved than the left ones. The left wrist showed a higher PDUS score than the right one, with a significant difference. The main limitations in this study were that the dominant hand definition was not taken in consideration, and the lack of a total score summation in each hand, which could have facilitated comparison of both sides (30).

Comparing the US5 scores between the two hands, the dominant hand was found to be significantly more affected, in particular in early RA cases. Significantly higher scores were found in the dominant hand for synovitis (both GS and PD) and erosions in early RA cases, and in the dominant hand for synovitis and tenosynovitis (PD only for both) in established RA cases. Furthermore, we found a significant relation between symmetry and seropositivity on one side and symmetry and activity on the other side. Moreover, erosions was related to disease duration and progression. These findings were comparable to the results from Zangger et al. (7). Their study focused on radiological evaluation of hand and foot symmetry in RA patients by modified Larsen score. They followed 2 unequal groups of patients longitudinally, one from England and another from Canada. RA patients were diagnosed according to ARA criteria. They examined all the MCPs, interphalangeal joints of the thumb, the four PIPs and the wrist joint in addition to foot joints. The overall asymmetry was found in $13 \%$ of the $1^{\text {st }}$ group and asymmetry was mainly in the MCPs and wrist joints. Progression to symmetry was detected in 58\% in the English group. In the second group, it was identified in approximately $30 \%$ of patients. The au- thors observed that the presence of RF did predict evolution to symmetry. Although the longitudinal pattern adds power to this study, it has some drawbacks. Two unequal groups of participants were considered, radiological evaluation was performed without US assessment, and finally there was no clinical evaluation for disease activity (31). Aga et al. studied two cohorts (32), the first including DMARD-naïve participants with early RA and disease duration $<2$ years; the second cohort had established RA with active disease. All were diagnosed according to the ACR/EULAR 2010 criteria. Both groups were subjected to US assessment of the hand, elbow and foot joints in addition to DAS28-ESR. The mean age in this study for both groups was around 50 (older than ours). Most of the participants in both groups had moderate disease activity. The aim of this study was to compare different US scores used for evaluation of RA patients. However, there was no clear indication about hand dominance and erosions, and the dominant hand was not compared with the non-dominant one. The conclusion of that study was that RA is not essentially a symmetrical disease, and that bilateral evaluation could provide additional information (32).

Backhaus et al. examined only the dominant hand with the US7 score (26). Furthermore, a 6-joint US assessment done by Perricone et al. (29) examined both hands, but included only the wrist and MCP 2 joints.

To our knowledge, our study is the first one to use US to evaluate the role of hand dominance in early and established RA.

RAI was always higher in the dominant hand in both groups, but this difference was more pronounced in group A in comparison to group $\mathrm{B}$, which also emphasizes the tendency towards symmetry over time. Adams et al. compared dominant and nondominant hands (5). In their study they included early RA patients, diagnosed by the ACR 1988 criteria, with a mean disease duration of 10 months. The mean age of the participants was 10 years older than that in our study. One advantage in this study is the clear definition and identifica- 
tion of hand dominance. They considered the Ritchie score, joint swelling, wrist mobility, ulnar deviation and hand grip. Wrist mobility was significantly impaired in the dominant side. Hand grip strength was less in the dominant hand with a non-significant difference. Ritchie score, swollen joint count and ulnar deviation were higher in the dominant hand with a non-significant difference. The drawbacks of this study comprise the lack of a comparative group of established RA patients, the absence of the 6 month cut-off between early and established RA, of disease activity evaluation as an inclusion criterion, and of US assessment. In addition, the frequency of a left handedness in their study was about $17 \%$, which differed greatly from patients in the present study.

Handgrip was stronger in the dominant hand in both groups. The difference was significant in group B and non-significant in group A. Björk et al. included in their study patients with a disease duration shorter than 1 year and followed them regularly for 5 years. They evaluated handgrip using the Grippit device. Their results showed that the dominant hand was always stronger, and, with disease progression, there was a manifest impairment of the handgrip (33). Their results were comparable to ours.

\section{CONCLUSIONS}

The dominant hand is more affected in early RA, and the disease becomes more symmetrical with its progression. This may suggest that an US evaluation of RA is performed on both hands in established RA, while in the early disease the examination of the dominant hand only may be satisfactory. In addition, protection of the dominant hand against mechanical stress in early RA might prevent disease progression in this side.

Acknowledgments: we thank Dr. Sarah Ohrndorf, Charite University Medicine, Berlin, Germany, for training the first author and for critical reading and editing of the article.
Conflict of interests: the authors declare no potential conflict of interests.

\section{REFERENCES}

1. Gibofsky A. Overview of epidemiology, pathophysiology, and diagnosis of rheumatoid arthritis. Am J Managed Care. 2012; 18: S295302.

2. Suresh E. Diagnosis of early rheumatoid arthritis: what the non-specialist needs to know. J R Soc Med. 2004; 97: 421-4.

3. Guillén Astete C, Boteanu A, Zea Mendoza A. Comparison of Prevalence of Synovitis by Ultrasound Assessment in Subjects Exposed or Not to Self-Reported Physical Overexertion: The Monday's Synovitis. Sci World J. 2014; 2014: 563981.

4. Mattingly P, Matheson J, Dickson RA. The distribution of radiological joint damage in the rheumatoid hand. Rheumatol. 1979; 18: 142-8.

5. Adams J, Burridge J, Hammond A, Cooper C. The effects of early rheumatoid arthritis on dominant and non-dominant hand impairment and function. Br J Hand Ther. 2005; 10: 93-7.

6. Koh JH, Jung SM, Lee JJ, et al. Radiographic structural damage is worse in the dominant than the non-dominant hand in individuals with early rheumatoid arthritis. PLoS One. 2015; 10: e0135409.

7. Zangger P, Keystone EC, Bogoch ER. Asymmetry of small joint involvement in rheumatoid arthritis: prevalence and tendency towards symmetry over time. Joint Bone Spine. 2005; 72: 241-7.

8. Hasselkus BR, Kshepakaran K, Safrit MJ. Handedness and hand joint changes in rheumatoid arthritis. Am J Occup Ther. 1981; 35: 705-10.

9. Pando JA, Duray P, Yarboro C, et al. Synovitis occurs in some clinically normal and asymptomatic joints in patients with early arthritis. J Rheumatol. 2000; 27: 1848-54.

10. Pfeil A, Hansch A, Lehmann G, et al. Impact of sex, age, body mass index and handedness on finger joint space width in patients with prolonged rheumatoid arthritis using computer-aided joint space analysis. Rheumatol Int. 2009; 29: 517-24.

11. Ohrndorf S, Backhaus M. Advances in sonographic scoring of rheumatoid arthritis. Ann the Rheum Dis. 2013; 72: ii69-75.

12. Ohrndorf S, Halbauer B, Martus P, et al. Detailed joint region analysis of the 7-joint ultrasound score: evaluation of an arthritis patient cohort over one year. Int J Rheumatol 2013;2013.

13. Scott D, Houssien D. Joint assessment in rheumatoid arthritis. Br J Rheumatol. 1996; 35: 14-8. 
14. Adams J. The effects of early rheumatoid arthritis on dominant and non-dominant hand grip ratio. Ann Rheum Dis. 2002; 61: 490.

15. Shiratori AP, Iop RdR, Borges Júnior NG, at al. Evaluation protocols of hand grip strength in individuals with rheumatoid arthritis: a systematic review. Rev Brasil Reumatol. 2014; 54: 140-7.

16. Cader MZ, Filer A, Hazlehurst J, et al. Performance of the 2010 ACR/EULAR criteria for rheumatoid arthritis: comparison with 1987 ACR criteria in a very early synovitis cohort. Ann Rheum Dis. 2011; 70: 1349.

17. Fransen J, Stucki G, van Riel PL. Rheumatoid arthritis measures: Disease Activity Score (DAS), Disease Activity Score-28 (DAS28), Rapid Assessment of Disease Activity in Rheumatology (RADAR), and Rheumatoid Arthritis Disease Activity Index (RADAI). Arthritis Care Res. 2003; 49: S214-24.

18. Singh JA, Saag KG, Bridges SL, et al. 2015 American College of Rheumatology Guideline for the Treatment of Rheumatoid Arthritis. Arthritis Rheum. 2016; 68: 1-26.

19. Backhaus M, Burmester G, Gerber T, et al. Guidelines for musculoskeletal ultrasound in rheumatology. Ann Rheum Dis. 2001; 60: 641-9.

20. Szkudlarek M, Court-Payen M, Jacobsen S, et al. Interobserver agreement in ultrasonography of the finger and toe joints in rheumatoid arthritis. Arthritis Rheum. 2003; 48: 955-62.

21. Scheel AK, Hermann KGA, Kahler E, et al. A novel ultrasonographic synovitis scoring system suitable for analyzing finger joint inflammation in rheumatoid arthritis. Arthritis Rheum. 2005; 52: 733-43.

22. Sokka T, Pincus T. Quantitative joint assessment in rheumatoid arthritis. Clin Exp Rheumatol. 2005; 23: S58-62.

23. Cooper C. The Effects of Early Rheumatoid Arthritis on Dominant and Non-dominant Hand Impairment and Function. Br J Hand Ther. 2005; 70.

24. España-Romero V, Ortega FB, Vicente-Rodríguez $\mathrm{G}$, et al. Elbow position affects handgrip strength in adolescents: validity and reliability of Jamar, DynEx, and TKK dynamometers. J Strength Cond Res. 2010; 24: 272-7.
25. Peolsson A, Hedlund R, Öberg B. Intra-and inter-tester reliability and reference values for hand strength. J Rehab Med. 2001; 33: 36-41.

26. Backhaus M, Ohrndorf S, Kellner H, et al. Evaluation of a novel 7-joint ultrasound score in daily rheumatologic practice: A pilot project. Arthritis Care Res. 2009; 61: 1194-201.

27. Naredo E, Rodriguez M, Campos C, et al. Validity, reproducibility, and responsiveness of a twelve-joint simplified power doppler ultrasonographic assessment of joint inflammation in rheumatoid arthritis. Arthritis Care Res. 2008; 59: 515-22.

28. Hammer HB, Sveinsson M, Kongtorp AK, Kvien TK. A 78-joints ultrasonographic assessment is associated with clinical assessments and is highly responsive to improvement in a longitudinal study of patients with rheumatoid arthritis starting adalimumab treatment. Ann Rheum Dis. 2010: annrheumdis 126995.

29. Perricone C, Ceccarelli F, Modesti M, et al. The 6-joint ultrasonographic assessment: a valid, sensitive-to-change and feasible method for evaluating joint inflammation in RA. Rheumatol. 2012; 51: 866-73.

30. Mendonca JA, Yazbek MA, Costallat BL, et al. The modified US7 score in the assessment of synovitis in early rheumatoid arthritis. Rev Bras Reumatol. 2014; 54: 287-94.

31. Zangger P, Keystone EC, Bogoch ER. Asymmetry of small joint involvement in rheumatoid arthritis: prevalence and tendency towards symmetry over time. Joint Bone Spine. 2005; 72: 241-7.

32. Aga A-B, Hammer HB, Olsen IC, et al. First step in the development of an ultrasound joint inflammation score for rheumatoid arthritis using a data-driven approach. Ann Rheum Dis. 2015: annrheumdis-2015-207572.

33. Björk MA, Thyberg IS, Skogh T, Gerdle BU. Hand function and activity limitation according to health assessment questionnaire in patients with rheumatoid arthritis and healthy referents: 5-year followup of predictors of activity limitation (The Swedish TIRA Project). J Rheum. 2007; 34: 296-302. 